\title{
Continuous Avalanche Segregation of Granular Mixtures in Thin Rotating Drums
}

\author{
Hernán A. Makse \\ Schlumberger-Doll Research, Old Quarry Road, Ridgefield, CT 0687 r
}

(Phys. Rev. Lett., 83, 3186 (1999))

\begin{abstract}
We study segregation of granular mixtures in the continuous avalanche regime (for frequencies above $\approx 1 \mathrm{rpm}$ ) in thin rotating drums using a continuum theory for surface flows of grains. The theory predicts profiles in agreement with experiments only when we consider a flux dependent velocity of flowing grains. We find the segregation of species of different size and surface properties, with the smallest and roughest grains being found preferentially at the center of the drum. For a wide difference between the species we find a complete segregation in agreement with experiments. In addition, we predict a transition to a smooth segregation regime - with an power-law decay of the concentrations as a function of radial coordinate - as the size ratio between the grains is decreased towards one.
\end{abstract}

Mixtures of grains differing in size, shape or density segregate in vibrated containers [1], in two and threedimensional rotating drums [2] 7], and by pouring the mixture into a heap [8.9]. For instance, axial band segregation appears in long rotating cylinders filled with mixtures of grains $[2$ 4], while radial segregation of granular mixtures is observed in thin $(\approx 10$ grain size) rotating drums: large grains are usually being found near the outer region of the drum, and small grains near the center of the drum [6,7].

Theoretically, the segregation of mixtures in rotating drums has been studied by Zik et al. [3] using a continuum approach based on mass conservation to treat band formation in 3-D drums. Recently, Khakhar et al. [5] predicted the concentration profiles in 2-D rotating drums filed with mixtures composed of equal-size grains of different density. However, there is no general analytical solution for segregation of grains in 2-D rotating drums in the case of mixtures of arbitrary size, and shape or friction properties. In this Letter, we study analytically this case focusing on the continuous avalanching regime, where a steady state is observed. Based on a continuum theory of surface flows of grains, we obtain predictions for the profile of the rolling species which agree quantitatively with experiments [2,5] when we take into account the velocity profile of the flowing grains.

We find that the small and rough grains occupy the center of the drum while the large and rounded grains segregate to the bottom of the drum. According to the size ratio and the different angles of repose of the species, there are two distinct regimes. For large size ratios our solution shows almost complete segregation of the mixture with a small region of mixing near half of the drum radius. The surface concentrations of grains behave exponentially on radial coordinate and segregation is characterized by a typical length scale inversely proportional to the size ratio between the grains. These findings agree with the experiments of Clément et al. [6]. On the other side, when the species differ slightly, we predict a slow power-law decay of the concentrations of grains at the surface, with no characteristic length scale associated to segregation.
In the continuous avalanching regime, grains on the free surface flow down steadily due to gravity forming a film of rolling species, while the bulk of the material rotates as a solid body. Thus, it is plausible to describe the system only by its surface properties 10 13.

We consider two local coarse-grained equivalent thicknesses of the species in the rolling phase $R_{i}(x, t)$, with $i=1,2$ [i.e. the total thickness of the rolling phase multiplied by the local volume fraction of the $i$ grains in the rolling phase at position $x$ on the surface of the bulk, Fig. 1(a)]. The total thickness of the rolling phase is then $R(x, t) \equiv R_{1}(x, t)+R_{2}(x, t)$. The static phase rotating as a bulk is described by the local angle of the surface height, $\theta(x, t) \equiv-\partial h(x, t) / \partial x[h(x, t)$ is the height], and the surface fraction of static grains, $\phi_{i}(x, t)$, of type $i$ at the surface of the bulk. The equations of motion for the rolling species are 12

$$
\frac{\partial R_{i}(x, t)}{\partial t}=v(x, t) \frac{\partial R_{i}}{\partial x}+\Gamma_{i}
$$

and the surface concentrations of static grains are given by

$$
\phi_{i}(x, t)\left(\frac{\partial h}{\partial t}-w x\right)=-\Gamma_{i},
$$

and $\phi_{1}+\phi_{2}=1$. Here $w$ is the rotation frequency of the drum. The downhill convection velocity of the rolling species $v(x, t)$ is usually taken as a constant [3, 10 13]. However, experiments on granular flows in drums, inclined chutes and sandpiles 2, 14, 15] indicates that the velocity varies linearly with the vertical position, $y$, of the particle in the flowing layer, i.e. $v(y)=2 \alpha y$, where $\alpha$ is the shear rate. In our continuum approach we take a height-averaged velocity dependence of the form [15]: $v(x, t)=1 / R(x, t) \int_{0}^{R} v(x, y, t) d y=\alpha R(x, t)$. We will show that the flux dependence of the velocity plays a crucial role in explaining the experimental results.

The interaction term $\Gamma_{i}$ takes into account the conversion of static grains into rolling grains and vice versa, and is [12], $[i=1,2,(j \neq i)]$

$$
\Gamma_{i} \equiv a_{i}(\theta) \phi_{i} R_{i}-b_{i}(\theta) R_{i}+x_{i}(\theta) \phi_{i} R_{j} .
$$


The interaction term is a function of the collision functions contributing to the rate processes. Amplification, $a_{i}(\theta)$ : an $i$ static grain is converted into a rolling grain due to a collision by a rolling grain $i$. Capture, $b_{i}(\theta)$ : an $i$ rolling grain is converted into a static grain. Crossamplification, $x_{i}(\theta)$ : the amplification of a $j$ static grain due to a collision by an $i$ rolling grain.

We consider the geometry of a two-dimensional rotating drum of radius $L[-L \leq x \leq L$, Fig. 11 (a)]. We study the continuous avalanche regime where steady state profiles are observed in the drum, and we seek a solution $\partial h(x) / \partial t=0, \partial R_{i}(x) / \partial t=0$, with boundary conditions $R_{i}(x=-L)=R_{i}(x=L)=0$.

The profile of the total thickness of the rolling phase $R(x)$ and the thickness of the rolling layer at the center of the drum $\lambda \equiv R(x=0)$ are obtained from (0.1):

$$
\begin{gathered}
R(x)=\sqrt{\frac{w}{\alpha}}\left(L^{2}-x^{2}\right)^{1 / 2}, \\
\lambda \equiv R(x=0)=\sqrt{\frac{w}{\alpha}} L
\end{gathered}
$$

The square root dependence in (0.3a) differs from the parabolic profile found in [11,3]. This latter result [11, 3] is obtained by assuming $v=$ const. Solving Eqs. $(0.1)$ for this case gives: $R(x)=w /(2 v)\left(L^{2}-x^{2}\right)$, and $\lambda=$ $w L^{2} /(2 v)$. In Fig. 2(a) we plot $\lambda(w)$ and compare with the digital photography experimental data of Khakhar et al. [5] for spherical sugar balls of $1.8 \mathrm{~mm}$ size, and the MRI experiments of Nakagawa et al. [2]. We clearly see that $\lambda(w)$ is consistent with a square root dependence on $w$ as in $0.3 \mathrm{~b}$ and not with a linear dependence on $w$ obtained by assuming $v=$ const 16 . In Fig. 2(b) we plot the theoretical prediction (0.3a) for $R(x)$, as well as the prediction obtained when $v=$ const, and compare with the experimental results of [5]. Since we use $\alpha=25 / \mathrm{s}$ obtained from Fig. 2(a), and $w=2 \pi f$ with $f=3 \mathrm{rpm}$ from the experiments, the comparisons in Fig. 2(b) are made without tuning of parameters. We again see an excellent agreement between experiment and theory only when the flux-dependent velocity is taken into account, while the prediction based on the assumption of a constant velocity profile gives lower values of $R(x)$ [17. This result is in agreement with the numerical analysis of [5] for simple shear flows.

To describe the system composed of a binary mixture of grains, we propose below a set of collision functions valid in the limiting cases of a wide and small difference between the species. We first treat the case where the difference in size and angles of repose is not too large (we will quantify this later). In this case the region of interest is concentrated in a small region around the angles of repose, a fact that allows us to linearize the collision functions in the vicinity of the angles of repose. We propose [Fig. 11 (b)]

$$
\begin{aligned}
a_{i}(\theta) \equiv x_{i}(\theta) & \equiv C+\gamma\left[\theta(x)-\theta_{i}\left(\phi_{j}\right)\right], \\
b_{i}(\theta) & \equiv C-\gamma\left[\theta(x)-\theta_{i}\left(\phi_{j}\right)\right] .
\end{aligned}
$$

Here, $\gamma \approx 23 / \mathrm{s} 15$ and $C$ are collision rates. In the continuous avalanche regime we have $w>\gamma \delta^{2} / 2$, where $\delta$ is the difference between the maximum angle of stability and the pure angle of repose of the species [11]. We note that the collision functions (0.4) differ from the ones proposed in [12] in the crucial fact that the generalized angle of repose, $\theta_{i}\left(\phi_{j}\right)$, of a rolling grain type $i$ depends on the composition of the surface $\phi_{j}[13]$ :

$$
\begin{aligned}
& \theta_{1}\left(\phi_{2}\right)=m \phi_{2}+\theta_{11} \\
& \theta_{2}\left(\phi_{2}\right)=m \phi_{2}+\theta_{21}=-m \phi_{1}+\theta_{22} .
\end{aligned}
$$

The limiting cases are $\theta_{i j}=\theta_{i}\left(\phi_{j}=1\right), m \equiv \theta_{12}-\theta_{11}=$ $\theta_{22}-\theta_{21}$, and the difference $\psi=\theta_{1}\left(\phi_{2}\right)-\theta_{2}\left(\phi_{2}\right)$ is independent of the concentration $\phi_{2}$, then $\psi=\theta_{11}-\theta_{21}=$ $\theta_{12}-\theta_{22}$.

The angle $\psi$ is proportional to the degree of difference in size and shape between the species, and therefore determines the degree of segregation; the larger $\psi$, the stronger the segregation. If the species 1 are the smallest then $\theta_{1}\left(\phi_{2}\right)>\theta_{2}\left(\phi_{2}\right)$ for any composition of the surface $\phi_{2}$, and also we have $\theta_{12}>\theta_{21}$. For mixtures of grains with different shapes or friction coefficients we have $\theta_{11} \neq \theta_{22}$, and $\theta_{12}=\theta_{21}$ if the species have the same size. If $\theta_{11}$ is the repose angle of the pure round species and $\theta_{22}$ is the repose angle of the pure rough species, then $\theta_{11}<\theta_{22}$. If the species have the same shape or friction coefficients then $\theta_{11}=\theta_{22}$. When the size ratio is close to one $\left(d_{2} / d_{1}<1.5\right.$ [8.9], $d_{i}$ size of grain $\left.i\right)$, the angle $\psi$ is small and then we linearize the collision functions as in (0.4).

Using (0.1b) and (0.4) we obtain the profile of the surface as a function of the rolling species

$$
\theta(x)-\theta_{1}\left(\phi_{2}\right)=\frac{-\psi R_{2}}{R}
$$

where we use that $d_{i} / \psi x \ll 1$. The equation for the rolling species of type 1 is obtained from Eqs. (0.1) and (0.6)

$$
\frac{\partial R_{1}(x)}{\partial x}=\frac{\partial R(x)}{\partial x}\left(1+\frac{\psi \gamma R_{2}}{C R}\right) \frac{R_{1}}{R^{2}}
$$

By setting $u(x) \equiv R_{1}(x) / R(x)$ we obtain $u^{\prime} /[u(1-u)]=$ $-\xi x /\left[L^{2}-x^{2}\right]$, where $\xi \equiv \gamma \psi / C$, and we arrive to a power-law form

$$
R_{1}(x) / R(x)=\frac{1}{1+A\left(L^{2}-x^{2}\right)^{-\xi}},
$$

where the power-law exponent $\xi \sim \psi \approx 0.2$ 15 depends on the degree of difference between the species, and $A=\left(\Phi_{2} / \Phi_{1}\right) L^{2 \xi}$ is an integration constant obtained from $(1 / 2 L) \int_{-L}^{L} \phi_{i}(x) d x=\Phi_{i}$, where $\Phi_{i}$ is the fraction of species $i$ at the surface (usually $\Phi_{i}=1 / 2$ ). The surface concentration profile shows a slow power-law decay 


$$
\phi_{1}(x)=u[1+\xi(1-u)] .
$$

Figure 3 shows the solutions obtained for this case (small $\psi$ ). The species 1 - the smallest grains or the roughest grains - are found preferentially at the center of the $\operatorname{drum}\left[\phi_{1}(x=0)>\phi_{2}(x=0)\right]$. The degree of segregation is small; the surface concentrations behave very smoothly as a function of the position in the drum.

According to (0.9), the exponent $\xi$, controls the intensity of segregation. Since $\xi \sim \psi \propto d_{2} / d_{1}$, then as we increase the size ratio, $\psi$ increases, and the linear approximation of the collision functions (0.4) breaks down. In this case, strong segregation effects act in the system. Segregation happens in the flowing layer as the large grains are observed to rise to the top of the rolling phase, while the small grains sink through the gaps left by the large grains; an effect known as kinematic sieving, free-surface segregation or percolation [18, 15]. Then the small grains are captured first near the center of the drum, since they interact with the surface before than the large grains. Thus, for large $\psi$ (in terms of size ratios $d_{2} / d_{1}>1.5$ [9]) the capture function of the large grains $b_{2}$ vanish near the angle of repose of the small type 1 species: the small rolling grains screens the interaction of the large rolling grains with the surface. We simulate this effect by considering the following non-linear collision functions 13] (valid for large $\psi$ ):

$$
\begin{aligned}
& a_{i}(\theta) \equiv \gamma \Pi\left[\theta(x)-\theta_{i}\left(\phi_{j}\right)\right] \\
& b_{i}(\theta) \equiv \gamma \Pi\left[\theta_{i}\left(\phi_{j}\right)-\theta(x)\right],
\end{aligned}
$$

where $\Pi[z]=0$, if $z<0$, and $\Pi[z]=z$, if $z \geq 0$ [Fig. 1 (c)].

Next we find the solutions for $x<0$ using (0.10) [19]. We consider that there are $R_{1}^{0}$ and $R_{2}^{0}$ rolling species of type 1 and 2 respectively at the center of the drum $\left(R_{1}^{0}+R_{2}^{0}=\sqrt{w / \alpha} L\right.$, and $\left.R_{2}^{0} /\left(R_{1}^{0}+R_{2}^{0}\right) \simeq \Phi_{2}\right)$. Since the collision functions are defined according to the value of $\theta(x)$ in comparison to $\theta_{i}\left(\phi_{2}\right)$, we divide the calculations in two regions: inner region, where $\theta_{2}\left(\phi_{2}\right)<\theta<\theta_{1}\left(\phi_{2}\right)$, and outer region, where $\theta<\theta_{2}\left(\phi_{2}\right)<\theta_{1}\left(\phi_{2}\right)$.

Inner Region. If $\theta_{2}<\theta<\theta_{1}$, we obtain from (0.1b) and (0.10): $\phi_{1}(x)=1, \phi_{2}(x)=0$. Then we obtain the profile of the rolling species using $(0.1)$

$$
R_{1}(x)=\sqrt{\frac{w}{\alpha}}\left(L^{2}-x^{2}\right)^{1 / 2}-R_{2}^{0}, \quad R_{2}(x)=R_{2}^{0} .
$$

The profile of the surface is: $\theta(x)-\theta_{11}=w x R_{1}(x) / \gamma$. This solution is valid when $\theta(x)>\theta_{2}\left(\phi_{1}=1\right)=$ $\theta_{21}$, or for $0>x>x_{m}=\left[\Phi_{2} \sqrt{w \alpha} / \gamma \psi-\right.$ $\left.\sqrt{1-\Phi_{2}^{2}+w \alpha /(\gamma \psi)^{2}}\right] L /\left[1+w \alpha /(\gamma \psi)^{2}\right]$.

The profile of the small rolling grains decays with the distance from the center as in the case of the total rolling species Eq. (0.3a), since the small grains are the only one effectively interacting with the surface due to percolation, so that it is a single species problem. Next, we find the solution of the problem at the lower end of the drum.
Outer Region. If $\theta<\theta_{2}<\theta_{1}\left(-L \leq x \leq x_{m}\right)$, using $(0.1)$ and (0.10) we obtain the equations for the rolling species

$$
\frac{\partial R_{1}(x)}{\partial x}=-\left(w x-\gamma \psi R_{2}\right) \frac{R_{1}}{\alpha R(x)^{2}} .
$$

To solve this equation we set $u \equiv R_{1}(x) / R(x)$, and obtain $u^{\prime} /[(1-u) u]=\gamma \psi /[\alpha R(x)]$ and the solution is

$$
R_{1}(x) / R(x)=\frac{1}{1+A \exp \left[-\left(x-x_{m}\right) / x_{s}\right]},
$$

where the characteristic length of segregation is $x_{s}=$ $\sqrt{w \alpha} L /(\gamma \psi) \sim d_{i} / \psi, A=\Phi_{2} / \Phi_{1}$ is an integration constant obtained by considering the continuity at $x=x_{m}$, and we have replaced $\exp \left\{\sin ^{-1}\left[\left(x-x_{m}\right) / L\right]\right\} \simeq \exp [(x-$ $\left.\left.x_{m}\right) / L\right]$. The concentration profiles is

$$
\phi_{1}(x)=\exp \left[\left(x-x_{m}\right) / x_{s}\right] .
$$

Figure 3 shows the theoretical profiles found for this case (large $\psi$ ). At the center of the drum we have total segregation of the small grains $\left[\phi_{1}(x=0)=1\right.$, $\left.\phi_{2}(x=0)=0\right]$. Then the concentration of small grains decays exponentially - with a region of mixing of characteristic length $x_{s}$. We also notice that in this case the profiles depend on $w\left[x_{s} \sim \sqrt{w}\right]$ while in the case of small $\psi$, the profiles (0.9) are independent of the rotation speed. Typical values of the phenomenological constants of the theory have been obtained experimentally in [15]: $\gamma \approx 23 / \mathrm{s}, \alpha \approx 25 / \mathrm{s}$ [see also Fig. 2(b)], and $\psi \approx 0.2$. Then we obtain $x_{s} \approx 1.6 \mathrm{~cm}$. These results are in quantitative agreement with experiments of Clément et al. [6], who found an exponential decay in the concentration of particles with a characteristic length of $\approx 1.7 \mathrm{~cm}$ for size ratio $d_{2} / d_{1}=1.5$. We also notice that $x_{s}$ decreases as $d_{2} / d_{1}$ increases, since $\psi \propto d_{2} / d_{1} ;$ a relationship found in the experiments of [6], too.

In sum, we show that the predictions based on the common assumption of a constant velocity profile of flowing grains are in error. The profiles predicted by the theory agree with experiments only when a height-averaged velocity profile is taken into account. If the difference between the species is wide, we find a sharp exponential segregation in agreement with experiments. It would be interesting to test experimentally the transition to the length scale-free segregation regime when $d_{2} / d_{1}$ is decreased below $\sim 1.5$. We believe that the present analysis has the potential to unify the mechanisms underlying segregation in different geometries such as silos and rotating drums. Our results are also valid for granular mixtures poured in 2-D silos: the different segregation regimes have been observed in [12, 13], and experimentally by Grasselli and Herrmann [9].

I wish to thank T. Boutreux for many discussions, and Collège de France for hospitality in initial stages of this work. 
[1] Physics of Dry Granular Matter, edited by H. J. Herrmann J.-P. Hovi, and S. Luding (Kluwer Academic Publishers, Dordrecht, 1998).

[2] M. Nakagawa, et al. Exp. Fluids 16, 54 (1993); M. Nakagawa, Chem. Eng. Sci. 49, 2544 (1994).

[3] O. Zik, et al., Phys. Rev. Lett. 73, 644 (1994).

[4] K. M. Hill and J. Kakalios, Phys. Rev. E 49, R3610 (1994).

[5] D. V. Khakhar, J. J. McCarthy, T. Shinbrot, and J. M. Ottino, Phys. Fluids 9, 31 (1997); D. V. Khakhar, J. J. McCarthy, and J. M. Ottino, Phys. Fluids 9, 3600 (1997).

[6] E. Clément, J. Rajchenbach, and J. Duran, Europhys. Lett. 30, 7 (1995).

[7] F. Cantelaube, and D. Bideau, Europhys. Lett. 30, 133 (1995); G. H. Ristow, Europhys. Lett. 28, 97 (1994); G. Baumann, I. M. Janosi, and D. E. Wolf, Phys. Rev. E 51, 1879 (1995).

[8] H. A. Makse, S. Havlin, P. R. King, and H. E. Stanley, Nature 386, 379 (1997).

[9] Y. Grasselli, and H. J. Herrmann, Granular Matter J. 1, 43 (1998).

[10] J.-P. Bouchaud, M. E. Cates, J. R. Prakash, and S. F. Edwards, Phys. Rev. Lett. 74, 1982 (1995).

[11] P.-G. de Gennes, in Powders 86 Grains 97 (eds. R. P. Behringer and J. Jenkins) (Balkema, Rotterdam, 1997).

[12] T. Boutreux and P.-G. de Gennes, J. Phys. I France 6, 1295 (1996).

[13] P. Cizeau, H. A. Makse, and H. E. Stanley, Phys. Rev. E 59, 4408 (1999).

[14] S. B. Savage, Adv. Appl. Mech. 24, 289 (1984); O. Pouliquen, and R. Gutfraind, Phys. Rev. E 53, 552 (1996). See also a recent theoretical study: A. Aradian, E. Raphaël, and P.-G. de Gennes, Phys. Rev. E 60, 2009 (1999).

[15] H. A. Makse, R. C. Ball, H. E. Stanley, and S. Warr, Phys. Rev. E 58, 3357 (1998).

[16] Deviations for $f>20 \mathrm{rpm}$ may indicate the limit of validity of the continuum avalanche regime.

[17] The profile based on $v=$ const is obtained using $v=19.4$ $\mathrm{cm} / \mathrm{s}$ at $x=0$ [5]. Even by rescaling the value of $v$ so that experiment and theory coincide at $R(x=0)$, we find that the shape of $R(x)$ obtained when $v=$ const does not fit the experiments.

[18] J. A. Drahun and J. Bridgwater, Powder Technol. 36, 39 (1983); S. B. Savage, and C. K. K. Lun, J. Fluid Mech. 189, 311 (1988).

[19] Profiles for $x>0$ are the same as $x<0$ since the grains perform a solid rotation without deforming.

FIG. 11(a) The thin rotating drum. (b) Linear approximation (solid lines) to the collision functions (dashed lines) when the difference between the species is small. (c) Non-linear approximation (solid lines) to the collision functions when the species differ appreciably. The collision functions are expected to be continuous (dashed lines), but we approximate them as discussed in the text.

FIG. 2(a) Theoretical prediction (0.3b) for $\lambda(w)$ and comparison with experiments of 2,5]. (b) $R(x)$ profiles from theory and experiment [5]. We also plot the prediction obtained with the assumption $v=$ const.
FIG. 3 Theoretical profiles $\phi_{i}(x)$ for small (dashed lines) and wide (solid lines) differences between the species (small and large $\psi$, respectively).
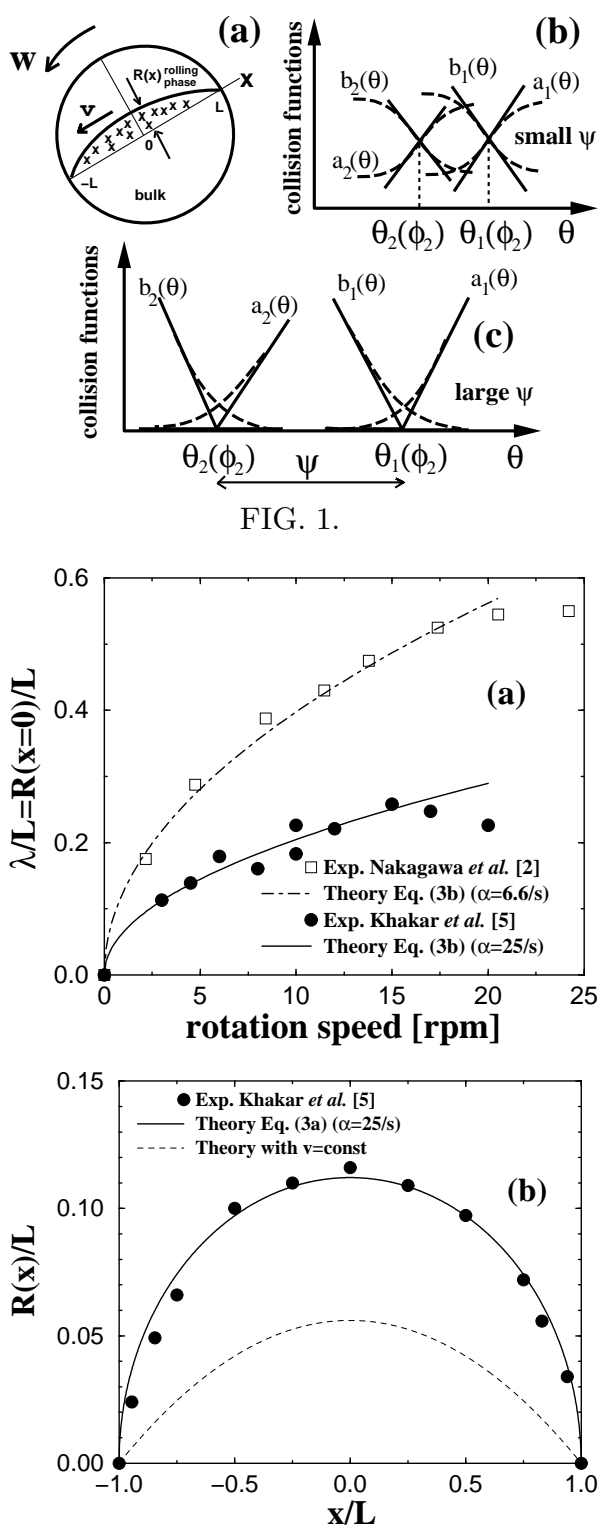

FIG. 2.

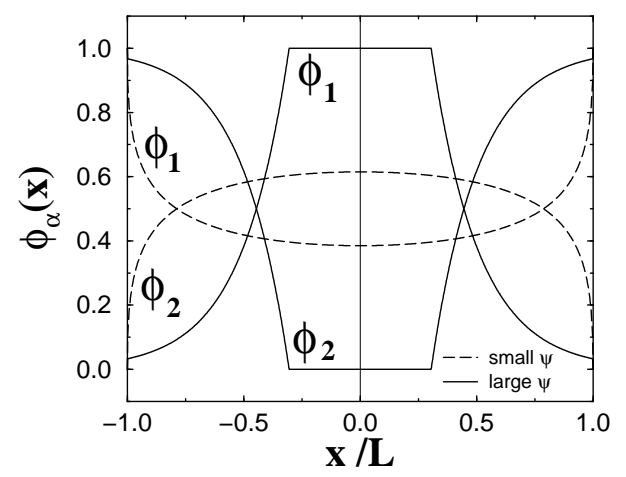

FIG. 3. 